\title{
Formulation and Evaluation of Tamoxifen Citrate Loaded Transdermal Reservoir Gel Drug Delivery Systems
}

\author{
Nagaraja SreeHarsha1,4,* Jagadeesh G Hiremath, Bimlesh Kumar Rawre ${ }^{2}$, Niranjan Puttaswamy ${ }^{5}$, \\ Bandar E Al-Dhubiab', Katharigatta N. Venugopala ${ }^{1,3}$, Sabah H Akrawi ${ }^{1}$, Girish Meravanige ${ }^{5}$, Mahesh \\ Attimarad ${ }^{1}$, Anroop B Nair ${ }^{1}$
}

1Department of Pharmaceutical Sciences, College of Clinical Pharmacy, King Faisal University, Al-Ahsa, SAUDI ARABIA. ${ }^{2}$ Department of Pharmaceutics, East West College of Pharmacy, Bengaluru, Karnataka, INDIA.

${ }^{3}$ Department of Biotechnology and Food Technology, Durban University of Technology, Durban, SOUTH AFRICA.

${ }^{4}$ Department of Pharmaceutics, Vidya Siri College of Pharmacy, Bangalore, Karnataka, INDIA.

${ }^{5}$ Department of Biomedical Sciences, College of Medicine, King Faisal University, Al-Ahsa, SAUDI ARABIA.

\begin{abstract}
Background: Successful treatment of cancer is yet remained as a challenging concern in public health. Transdermal delivery of hormonal therapy offers several advantages over the oral route associated with potential side effects. Methods: The present investigation preparation and screening of rate controlling membranes using Eudragit release liners of RS 100, hydroxyl propyl methyl cellulose K4M (HPMC K4M) and ethyl cellulose by plate casting method and their physicochemical characterization. The prepared release liners were subjected for preformualtion studies (thickness, weight variation, moisture uptake, folding endurance and moisture loss). Tamoxifen citrate is an ideal molecule for the transdermal reservoir for the preparation of gel dosage form due its dosage regimen. Results: The main investigation, topical gel TC was prepared using HPMC K4M and Carbopol 934 as gelling agents in different proportions with Dimethyl Sulfoxide (DMSO) as a penetration enhancer. TC gels were subjected for the physicochemical parameters such as $\mathrm{pH}$, viscosity, spreadability. Conclusion: The TC gel diffusion was conducted by using rate controlling membranes (release liner). The TC gel skin permeation was investigated by using excised rat skin as a barrier.
\end{abstract}

Key words: Transdermal reservoir, Gel, Tamoxifen Citrate (TC), Penetration Enhancer.

\section{INTRODUCTION}

After decades of great improvement in strategies of cancer treatment, it is still considered a great challenging disease for health professionals. Hormonal chemotherapy of cancer is an efficient method of cancer treatment in hormone-responsive breast cancer patients. Emerged in the early $19^{\text {th }}$ century, hormonal chemotherapy, such as removing patient's adrenal glands (bilateral adrenalectomy), became a common practice in breast cancer treatment, as the only option of therapy. Soon after, tamoxifen and further aromatase inhibitors were discovered and substituted the organ dissection approach in breast cancer treatment protocols. Tamoxi- fen Citrate (TC) is an aromatase inhibitor prodrug which can be metabolized in the liver and became a potent Estrogen Receptor (ER) ligand. TC is one of the first lines of therapy for treating hormone-responsive breast cancer in pre- and post-menopausal women. ${ }^{1}$

The delivery of hormones in cancer hormonal chemotherapy can be accomplished by several administration routes, mainly categorized into parenteral and non-parenteral routes. Transdermal drug delivery route is one of the favorite non-parenteral routes of administrations in this case. A typical Transdermal Drug Delivery Sys-
Submission Date: 14-05-2019; Revision Date: 29-06-2019; Accepted Date: 10-08-2019

DOI: 10.5530/ijper.53.4s.155 Correspondence:

Dr. Nagaraja SreeHarsha, Department of Pharmaceutical Sciences, College of Clinical Pharmacy, King Faisal University-31982, Al-Ahsa, SAUDI ARABIA.

Phone: : +96 6535485322

E-mail: sharsha@kfu.edu.sa

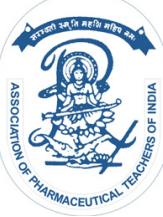

www.ijper.org 
tem (TDDS) consists of an adhesive patch containing a drug reservoir (commonly a gel system) and a ratecontrolling membrane made of polymers. This system is applied on the skin and via that a controlled and sustained drug release pattern can be achieved to the skin surface. In general, TDDS systems have many specific advantages over other delivery approaches, of note, the improved patient compliance due to their non-invasive nature, negligible side effects and less chance of drug overdosing in patients. A number of FDA approved transdermal products have demonstrated a successful application of TDDS, for instance, Transderm-Nitro ${ }^{\text {TM }}$, Estraderm $^{\text {TM }}$, Duragesic ${ }^{\text {TM }}$, D-TRANS ${ }^{\text {TM }}$, Androderm ${ }^{\text {TM }}$ and Catapres-TTS ${ }^{\text {TM }}{ }^{2}$ An optimized TDDS can release its embedded active molecules with a narrow therapeutic index in a very controlled fashion. ${ }^{3-5}$ However, to reach a successful therapeutic outcome, the released therapeutic agent should pass a very complicated process from the skin surface to the systemic blood circulation. In this process, several steps have major effects on the overall bioavailability of the applied drug, such as physicochemical properties of the active ingredient (dissolution behavior, partition coefficient, etc.) and its passive diffusion through different layers of skin, such as epidermis and dermis. ${ }^{6}$ The general method for the preparation of a gel as a reservoir of a TDDS is by combining the hydrophilic and hydrophobic polymers and physically or chemically cross-links them to have a viscoelastic gel structure. Therefore, an important criterion to design an efficient TDDS is the selection of the appropriate embedding polymer. Several gel-forming polymers have been reported in the literature for TDDS preparation. Among them, hydroxypropyl methylcellulose (eg., Methocel K4M, 4000cP and Methocel K100M, 75000cp) and Carbopols (eg., Carbopol ${ }^{\circledR}$ 934) are attracted more attention, due to their suitable biocompatibility and ease of application. ${ }^{7,8}$ For instance, Carbopols are a group of high molecular weight carboxyvinyl, homo-and copolymers of acrylic acid which are the suitable hydrogel-forming polymers. If the polymeric gels are cross-linked by polyethers, such as allyl sucrose, the resulting polymeric gel behaves like a non-Newtonian fluid. Examples of these hydrogels were used in the preparation of typical reservoir TDDS formulations. ${ }^{9}$

Next step of TDDS fabrication is to control the release of the drug from the reservoir. Various polymers are reported in the literature as a rate-controlling membrane for developing a reservoir TDDS. For instance, cellulose derivatives, chitosan, carrageenan, polyacrylates, polyvinyl alcohol, polyvinylpyrrolidone and silicones are among the most used membrane structures which were reported in the past. ${ }^{6,10}$

Eudragit RL100 and Eudragit RS100, both are copolymers of ethyl acrylate and methyl methacrylate and have a low content of methacrylic acid ester with some quaternary ammonium groups in their molecular structures. While technically insoluble in water, the presence of quaternary ammonium groups as a salt causes these polymers to be very little (RS100) to highly (RL100) permeable to the water. ${ }^{7,8}$ This unique characteristic enables researchers to customize the release profile of the drugs in TDDS by a combination of different ratios of RL and RS grades of Eudragit in the rate-controlling membrane. Additionally, there are several reports on the controlled release of drugs by TDDS and other dosage forms by using Ethyl Cellulose (EC) and hydroxypropyl methylcellulose K4M (HPMC K4M). Additionally, the hydrophobic nature of HPMC K4M and ethyl cellulose were investigated for the preparation of rate-controlling membrane for the release of TC. ${ }^{11}$

Another important factor in TDDS preparation is plasticizer. Plasticizers are commonly used in the film forming systems to impart appropriate flexibility to the film and improve the tensile strength of the prepared film. The plasticizer should be compatible with the applied polymers and should have low skin permeability. ${ }^{11,12}$ As an example of plasticizer, dibutyl phthalate (DBP) $10-30 \%(\mathrm{w} / \mathrm{w})$ was investigated in a report for evaluation of its effect on the drug diffusivity which is a critical factor for developing the controlled release membranes or films.

In general, the outer layer of the skin epidermis- Stratum Corneum (SC) is the rate-limiting barrier for the drug for passive diffusion through skin. SC mainly consists of keratinized and lipidic dead cells with no active transport capability. Transport of substances across this layer is solely due to the passive diffusion. To enhance the drug permeation through the skin, penetration enhancers are often used which can increase the drug diffusivity or drug partitioning from the formulation or to skin layers, respectively. ${ }^{13,14}$ The mechanism of action of penetration enhancers is suggested to be by fluidizing or disordering the lipids and proteins of SC structures..$^{15}$ However, the exact mechanism of action of these penetration enhancers is not clear. ${ }^{16,17}$ For example, it has been reported that when Dimethyl Sulfoxide (DMSO) was used as a penetration enhancer it shows three different mechanisms of action on the cell membrane based on its concentration. At the lower concentrations (2.5$7.5 \mathrm{~mol} \%$ ), it induces the expansion and thinning of the lipid bilayer, thereby increasing the fluidity of the hydrophobic region of the membrane. At the middle range of 
concentrations (10-25 mol \%), it induces the formation of transient "water pores" in the cell membrane, consequently increases the fluidity of the bilayer. ${ }^{18}$ The reorientation of lipid head groups minimizes the free energy of the system, which results in pore formation in the skin surface. ${ }^{15-17}$ However, at the higher concentrations (25-100 mol\%), DMSO can extract lipid molecules and disintegrate the lipid layer in SC. ${ }^{19-21}$

The purpose of this study was to design a transdermal delivery system of TC for hormonal therapy of breast cancer. For that, various TDDS formulations were developed and tested by different release ratecontrolling membranes and reservoir hydrogels using a combination of appropriate polymers and excipients. In this work, we find out a rate-controlling membrane, consisting of the appropriate composition of Eudragit RL100 and RS100, HPMC K4M or EC. At the same time, we prepared a reservoir gel that can provide the required physicochemical properties (increased viscosity for optimal skin application and improved skin penetration) for an effective transdermal formulation by using Methocel and Carbopol ${ }^{\circledR} 934$ and/or their combination. We also used a relatively vast screening method in vitro to find out the suitably performing membrane and reservoir combinations in TDDS for transdermal hormonal therapy of breast cancer.

\section{MATERIALS AND METHODS}

\section{Materials}

Tamoxifen citrate and Eudragits RL, RS100 was a purchased from Sigma Aldrich (St. Louis, USA). HPMC K4M and Methocel K100M were also gift samples from Rolex Chemical Industry, India, Ethyl cellulose and EC Carbopol ${ }^{\circledR} 934$ were procured from Titan Biotech Ltd, India. ScotchPak ${ }^{\text {TM }} 1109$ backing membrane was obtained from 3M Drug Delivery Systems, Bangalore India. Other solvents and reagents used were of analytical reagent grade.

\section{Preparation of release rate liners}

The series of preformulation screening study was adopted for the selection of appropriate quantity of polymers, additives and solvents. Sixty release liners were prepared by using low to highest concentration of polymers, additives and solvents and check the physical integrity tests the release liners. Finally (12) release liners were screened out and the same method was reproduced in triplicate and standardized. The release liners were prepared by solvent casting technique according reported methods and modified accordingly to our research laboratory. ${ }^{22-25}$ In brief, the polymeric solutions were prepared by using appropriate solvents, to the polymeric solution the dibutyl phthalate (DBH) 10-30\% $(\mathrm{w} / \mathrm{w})$ of polymer was added as a plasticizer to enhance the plasticity and mixed the solution homogenously for a period of 15 min by using closed vials multi vortexer (Remi, Bangalore, India). The viscous solutions of different concentration were transferred to ptri dish plates using aluminium foil as backing membrane with an area of $63.59 \mathrm{~cm}^{2}$ inverted glass funnel with cotton plug was placed over the plates to prevent the air and the plates were kept aside for drying at room temperature for 24 hr. After drying the release liners were peeled from Petridis plates and closed both sides by using aluminium foil, with having same area and preserved in desiccators for further studies. The formulae for the release liner are given in Table 1.

\section{Evaluation of release rate liners}

The prepared release membranes were subjected to various physicochemical evaluations, including visual properties (any cracks or ruptures), measuring of the thickness, weight uniformity, extent of moisture absorption, folding endurance and tensile strength evaluations. The thickness of the prepared films was measured for each batch, using digital verniermicrometer (Mitoyoto, Japan) at different regions and the mean value of each sample was determined. Each individual film was initially weighed (Precision Digital Instrument, India) and then the percentage of moisture absorption was determined by placing the films for $72 \mathrm{~h}$ in a desiccators where a constant $84 \%$ Relative Humidity [RH] was provided by using a saturated sodium chloride solution. During this time, the weight of the films was periodically measured. The difference between the final and the initial weight of the films was considered as the extent of moisture absorbance when the weight variation of the film reached the plateau.

The folding endurance of the films was determined by cutting a $2 \times 2 \mathrm{~cm}$ section of each prepared batch and then repeatedly folded until it was broken. The number of times the film could be folded until either to break or to develop visible cracks was recorded for each sample as the durability of the film. The tensile strength usually measures the force required to break apart a film membrane. The tensile strength of the prepared film was determined by using a Tensiometer (Erection and instrumentation Co., Ahmedabad, India). A $2 \times 2 \mathrm{~cm}$ film was fixed between the lower and upper cell grips and the force then was applied on the upper grip until the film was broken. The applied force was recorded and the Young Modulus factor was determined for each 
sample. The detailed measured parameters of the prepared films were presented thoroughly in Table 2.

\section{Preparation of reservoir gel}

To cast the reservoir gel of the TDDS, first the drug (100mg of TC) was completely dissolved in $5 \mathrm{ml}$ of methanol, then DMSO was added (as a penetration enhancer) to the methanolic drug solutions to make $10-30 \% \mathrm{w} / \mathrm{v}$ of DMSO mixture. Next, $100 \mu \mathrm{L}$ of Tween 80 was added as a surfactant to maintain the uniform phase of the drug mixture. After that, Carbopol 934, HPMC-K-100M or combinations of their different ratios were used as the gelling agents. For that, first $1 \mathrm{~g}$ of each polymer or a mixture of them was mixed with $50 \mathrm{~mL}$ deionized water. In this process, the sieved polymer powder was gradually added to the solvent over 30 meanwhile mixing by a tiny three-blade mechanical stirrer (Remi Pvt Ltd., India) at $150 \mathrm{rpm}$. Next, the $\mathrm{pH}$ value was adjusted at 7.4 and then the mixture was covered with aluminium foil and continuously stirred at the same speed for further $24 \mathrm{hr}$. During this time, the polymer mixture was evaluated regularly to be completely hydrated and dispersed. Finally, the initially prepared drug solutions were gradually added into the hydrogel phase and allowed to be stirred for $2 \mathrm{hr}$ to form a uniform gel. The compositions of the prepared TC containing gel formulations are shown in Table 3.

\section{In vitro characterization of reservoir gel}

The viscosity of the prepared gel was performed using Brooke Field Viscometer (Bob and Cup type, Commerce Boulevard, Middleboro, MA 02346-1031 USA). The steady shear measurements were recorded at $25^{\circ} \mathrm{C}$ at 50 and $100 \mathrm{rpm}$ using spindle number 5 , accurately 30 gm of gel was transferred to cup holding cylindrical steel tube and the viscosity of the hydrogel were recorded, experiments were conducted in triplicate. The $\mathrm{pH}$ of the gel was measured using (HM digital Hydrometer-80 USA). The gel spread ability of the TC containing 0.5 $\mathrm{g}$ of gel was placed with premarked $1 \mathrm{~cm}$ diameter of a circle on a glass plate, over which a second glass plate was placed. The TC gel $500 \mathrm{~g}$ was introduced on the upper glass plate for 8 min. ${ }^{23}$ The increase in the diameter due to spreading of the gel was determined; the experiment was performed triplicate average vales were reported results are shown in Table 2 . The uniformity of the drug content of the casted gel was determined known quantity of TC gel was dissolved in dichloromethane: acetone 1:1 ratio, filtered the solutions using $0.22 \mu \mathrm{m}$ filter, organic phase was evaporated and serial dilution were made with mobile phase and tamoxifen citrate was determined by reported method in brief, Chromatographic separations were achieved with a Vanquish $^{\mathrm{TM}}$ C-8 column ( $\left.4.8 \times 250 \mathrm{~mm}, 5 \mu \mathrm{m}\right)$ and a

\begin{tabular}{|c|c|c|c|c|c|c|c|c|c|c|c|c|}
\hline Components & $\mathbf{R} 1$ & $\mathbf{R 2}$ & R3 & R4 & R5 & R6 & R7 & R8 & R9 & R10 & R11 & R12 \\
\hline RL 100 & 200 & 400 & 600 & & & & & & & & & \\
\hline RS 100 & & & & 200 & 400 & 600 & & & & & & \\
\hline HPMC K4M & & & & & & & 200 & 400 & 600 & & & \\
\hline EC & & & & & & & & & & 200 & 400 & 600 \\
\hline$\%$ DBT $(w / w)(m g)$ & 20 & 80 & 180 & 20 & 80 & 180 & 20 & 80 & 180 & 20 & 80 & 180 \\
\hline Acetone $(\mathrm{ml})$ & 5 & 5 & 10 & 5 & 5 & 10 & & & & & & \\
\hline Methanol (ml) & 5 & 10 & 10 & 5 & 10 & 10 & 5 & 10 & 10 & 3 & 6 & 9 \\
\hline Chloroform (ml) & & & & & & & & & & 7 & 9 & 11 \\
\hline Isopropyl alcohol (ml) & & & & & & & 3 & 3 & 5 & & & \\
\hline Dichloromethane (ml) & & & & & & & 2 & 2 & 5 & & & \\
\hline
\end{tabular}

\begin{tabular}{|c|c|c|c|c|c|c|c|c|c|}
\hline \multicolumn{10}{|c|}{ Table 2: Composition of TC containing gel. } \\
\hline Components & TC1 & TC2 & TC3 & TC4 & TC5 & TC6 & TC7 & TC8 & TC9 \\
\hline TC (mg) & 100 & 100 & 100 & 100 & 100 & 100 & 100 & 100 & 100 \\
\hline HPMC-K-100 (mg) & 1000 & & 250 & 1000 & & 250 & 1000 & & 250 \\
\hline Carbopol 934 (mg) & - & 1000 & 750 & & 1000 & 750 & - & 1000 & 750 \\
\hline Tween 80 (0. 2\% w/v of drug) & 0.1 & 0.1 & 0.1 & 0.1 & 0.1 & 0.1 & 0.1 & 0.1 & 0.1 \\
\hline DMSO (\% w/w) & 10 & 10 & 10 & 15 & 15 & 15 & 20 & 20 & 20 \\
\hline Methanol (ml) & 5.8 & 5.8 & 5.8 & 5.8 & 5.8 & 5.8 & 5.8 & 5.8 & 5.8 \\
\hline Water (ml) QS & 50 & 50 & 50 & 50 & 50 & 50 & 50 & 50 & 50 \\
\hline
\end{tabular}


Vanquish ${ }^{\mathrm{TM}}$ C-8 guard column cartridge $(4.0 \times 3.0 \mathrm{~mm}$, $5 \mu \mathrm{m})$. A mobile phase consisting of methanol, water and TEA (90:10:0.1\% v/v) was passed through a $0.22-$ $\mu \mathrm{m}$ membrane filter and degassed by ultrasonication in vacuum before use. The analysis was performed at the flow rate of $1 \mathrm{~mL} / \mathrm{min}$ with a UV detector at $265 \mathrm{~nm}$ and the sensitivity was 1.0 absorbance unit force per sec. $^{23,24}$ The in vitro drug diffusion study was conducted by using Kesiery-Chein Diffusion cell. The acceptor compartment of the applied diffusion cell had a volume of $25 \mathrm{~mL}$ with an effective surface area of $2.8 \mathrm{~cm}^{2}$ and filled with $25 \mathrm{~mL}$ of PBS $\mathrm{pH}$ 7.4. The temperature of the cells was kept constant at $37 \pm 1{ }^{\circ} \mathrm{C}$ and the acceptor compartment solution was kept stirring at $250 \mathrm{rpm}$ with magnetic bead during the experiment. To test the diffusion of TC containing gel, we used the prepared R1-R12 rate-controlling membranes. For that, first $1 \mathrm{~g}$ of each gel sample was uniformly spread on the ratecontrolling membranes (with $3.0 \mathrm{~cm}^{2}$ diameter effective area would be $2.8 \mathrm{~cm}^{2}$ ) and then the surface was covered by ScotchPak ${ }^{\text {TM }} 1109$ backing membrane and placed between donor and acceptor compartments. The $2 \mathrm{~mL}$ of samples were withdrawn from the acceptor compartment at the specific time intervals with a replacement of an equal volume of fresh PBS solvent to the acceptor compartment. Next, the samples were analyzed by the above mentioned optimized HPLC method.

We also tested the diffusion of the drug with the extracted abdominal skin of the Female Sprague Dawley rats using the procedure reported previously. ${ }^{25}$ Briefly, the subcutaneous tissues and fats of the extracted skin were carefully removed, noticing to not damage the epidermis layer. The selected gel formulations (TC3, TC6 and TC9) and the rate-controlling membranes (R3, R6 and R12) were investigated in this part, using Keshary-
Chien type diffusion cells as described above with the exception that the rate-controlling membrane was placed over the rat's skin. Equivalent $10 \mathrm{mg}$ TC containing gel $(1 \mathrm{gm})$ was spreaded to the release liner and then the ScotchPak ${ }^{\text {TM }} 1109$ backing membrane was used to cover the rate-controlling membrane on the top of the drug-gel complex. ${ }^{26}$ This assembly was placed on the skin were mounted on diffusion cell across hairless rat skin the experiment procedure is similar to in vitro skin permeation study. The withdrawn $2 \mathrm{~mL}$ samples were similarly analyzed by HPLC method. The cumulative amount of drug diffused per unit surface area of the skin was then plotted versus time. The slope of the linear portion of the plot was calculated as flux, Jss $(\mu \mathrm{g} /$ $\mathrm{cm}^{2} / \mathrm{hr}$ ) and the permeability coefficient was calculated using Eq. 1: $\mathrm{Kp}=J \mathrm{ss} / \mathrm{C}^{26,27}$ For this section, the protocols of animal handling procedures were performed in accordance with the CPCSEA guidelines.

\section{RESULTS AND DISCUSSION}

\section{Formulations aspects}

In this study, we tried to prepare the rate-controlling membranes and appropriate hydrogels for controlled/ sustained delivery of TC loaded TDDS for hormonal therapy of breast cancer.

A reservoir-type TDDS is generally composed of a drug reservoir (in solution or hydrogel formulations) located between an impermeable backing layer and a rate-controlling membrane which will be in contact to the skin surface of the patients. The rate-controlling membrane can be either a microporous or a nonporous polymeric membrane. Additionally, a pressure sensitive adhesive polymer is usually applied on the external surface of the

\begin{tabular}{|c|c|c|c|c|c|}
\hline \multicolumn{5}{|c|}{ Table 3: Evaluation of rate controlling release liners plasticized with DBT. } \\
\hline Formulation Code & $\begin{array}{c}\text { Weight uniformity } \\
(\mathbf{m g})\end{array}$ & $\begin{array}{c}\text { Tensile } \\
\text { strength N/mm }\end{array}$ & Thickness (mm) & Folding endurance & $\begin{array}{c}\text { Moisture up take (\%) } \\
\mathbf{7 5} \text { RH }\end{array}$ \\
\hline R1 & $0.28 \pm 0.01$ & $0.64 \pm 0.22$ & $0.14 \pm 0.01$ & $67 \pm 6.5$ & $3.12 \pm 1.5$ \\
\hline R2 & $0.64 \pm 0.01$ & $0.56 \pm 0.12$ & $0.19 \pm 0.02$ & $86 \pm 3.8$ & $5.42 \pm 3.1$ \\
\hline R3 & $0.92 \pm 0.02$ & $0.34 \pm 0.16$ & $0.22 \pm 0.03$ & $120 \pm 4.6$ & $4.12 \pm 4.1$ \\
\hline R4 & $0.29 \pm 0.01$ & $0.61 \pm 0.09$ & $0.14 \pm 0.02$ & $58 \pm 3.6$ & $3.9 \pm 2.10$ \\
\hline R5 & $0.66 \pm 0.03$ & $0.44 \pm 0.32$ & $0.18 \pm 0.04$ & $86 \pm 2.8$ & $5.42 \pm 5.2$ \\
\hline R6 & $0.91 \pm 0.01$ & $0.36 \pm 0.19$ & $0.23 \pm 0.02$ & $126 \pm 3.7$ & $5.14 \pm 2.9$ \\
\hline R7 & $0.32 \pm 0.04$ & $0.66 \pm 0.42$ & $0.15 \pm 0.01$ & $62 \pm 3.9$ & $4.13 \pm 0.9$ \\
\hline R8 & $0.70 \pm 0.04$ & $0.56 \pm 0.56$ & $0.20 \pm 0.02$ & $88 \pm 1.2$ & $5.19 \pm 1.1$ \\
\hline R9 & $0.93 \pm 0.04$ & $0.28 \pm 0.07$ & $0.24 \pm 0.01$ & $132 \pm 1.8$ & $5.12 \pm 3.1$ \\
\hline R10 & $0.31 \pm 0.06$ & $0.59 \pm 0.08$ & $0.13 \pm 0.04$ & $74 \pm 2.4$ & $6.52 \pm 1.4$ \\
\hline R11 & $0.68 \pm 0.01$ & $0.41 \pm 0.12$ & $0.19 \pm 0.03$ & $90 \pm 2.6$ & $7.32 \pm 3.1$ \\
\hline R12 & $0.96 \pm 0.04$ & $0.29 \pm 0.45$ & $0.23 \pm 0.01$ & $146 \pm 1.2$ & $7.84 \pm 2.4$ \\
\hline
\end{tabular}


rate-controlling membrane to provide close contact of TDDS with the skin surface. ${ }^{26}$

\section{Release liners (rate controlling membranes) studies}

In the present study, efforts have made to prepare rate controlling membranes for control/sustained release of tamoxifen citrate loaded hydrophilic and hydrophobic gel were prepared and characterized. Eudragit release linear, RS 100, K4M and EC have good film forming property. The casting method on mercury surface was found to be satisfactory to get thin and transparent or slightly translucent release liners (release linear). Release linear plasticized with 10\% DBP were slightly brittle and tougher and those with 20\% and 30\% DBP were soft and also found to be optimum with respect to smoothness, flexibility and transparency. Tamoxifen citrate dosage $10-40 \mathrm{mg}$ daily administration in the form of tablets for a period 5 years. Based on the pharmacological/desired therapeutic level of drug was calculated. $1 \mathrm{gm}$ of gel equivalent $10 \mathrm{mg} / 2.8 \mathrm{~cm}^{2}$ which can release a drug for period of $24 \mathrm{~h}$. The release linear plasticized with DBP possessed high tensile strength and variations in folding endurance with respect to the concentration of DBP and additives. The release liners of R3, R9 and R12 films showed the low tensile strength between $0.34 \pm 0.16-0.29 \pm 0.45 \mathrm{~N} / \mathrm{mm} .^{2}$ It indicates that plasticizer molecules are interacting with the forces held together by polymer chains enhancing to softening of the polymer nature. ${ }^{28}$ This phenomenon also correlating to the folding endurance of the films, as the DBP concentration was increased the release linear folding endurance was increase, the DBP $30 \% \mathrm{w} / \mathrm{w}$ release linear showed $120 \pm 0.96-146 \pm 1.2$ compared release linear prepared with10-20\% w/w of DBT.

All the prepared batches of transdermal patches showed the thickness variations ranged from $0.14 \pm 0.01$ to $0.23 \pm 0.01 \mathrm{~mm}$ as shown in Table 3. R6, R9 and R12 films showed a higher thickness ranging from $0.29 \pm 0.01$ to $0.96 \pm 0.04$, which may indicate that the low solubility of the polymer in the selected solvents render uneven distribution of the polymer layer in this case. Table 3 shows that the percentage of the absorbed moisture ranged between $3.12 \pm 1.5$ to $7.84 \pm 2.4$ (at $75 \% \mathrm{RH})$ for formulations of R6, R9, R10, R12 membranes. This indicates that due to the difference in the composition of the films.

\section{Characterization of tamoxifen loaded reservoir transdermal delivery systems.}

To characterize the reservoir part of the TDDS, we prepared several drug-containing physical gels using hydrophilic and hydrophobic polymers. Physical gels have different macroscopic properties because of their different elastic, viscous, plastic and thixotropic characteristics. ${ }^{29}$ The HPMC-K100 gel showed a moderately higher range of viscosity values from $900-1000 \mathrm{cp}$. At the same time, the range of viscosities of Carbopol-934 was the highest ranging from 1800-1900cp. The observed variations in viscosity can be explained by the polymer nature and the water swelling capability of the polymers. HPMC-K100 is a freely water-soluble polymer. Moreover, our study indicated a non-Newtonian (viscoelastic) ${ }^{30}$ characteristics for these applied polymers. Figure 1 shows the Scanning Electron Microscopy (SEM) images of TC3 and TC9 hydrogel formulations. SEM of HPMC K100 formulation showed that the drug was embedded as crystals in the polymer (gel) matrix. SEM image of TC9 was observed as a plane or smooth matrix gel structure and it seemed that no drug crystals could be observed in the matrix. This might be because that drug was embedded in the amorphous form in the gel matrix probably due to the presence of DMSO in the formulations. Besides, the polymer/drug mutual interactions can a significant effect on the final polymorphism of the drug in the formulations.

\section{In vitro Tamoxifen citrate permeation studies}

An in vitro permeation study was carried out by using Keshary-Chien diffusion cell to evaluate R1 to R12 ratecontrolling membrane formulations. Formulations of TC3, TC5, TC6 T10, TC11 and TC12 showed 60-78\% of cumulative amount of drug permeated through release liners of R3, R6, R9 and R12 at the end of 24h. It indicates other formulations had low concentration of polymer, DBT and DMSO hence the brittle liners have hindered the formation of micro pores on the surface of the release liners. The highest cumulative amount of drug permeated or diffused from formulation TC3, TC6 and TC9 (from in vitro release data) was the concentration of hydrophilic and lipophilic combination of polymers. This diffusion performed across the release liner of R3, R9 and R12.. ${ }^{29,31}$ Results are shown in Figure 2-4. Figure 5 and 6 shows the order of highest $J$. Flux and permeability coefficient was found in formulations TC3>TC6 $>$ TC9 with $(\mathrm{R} 3>\mathrm{R} 9>\mathrm{R} 12$ release liners) this attributed due the concentration effect of penetration enhancer DMSO between 10 to $20 \% \mathrm{w} / \mathrm{w}$ indicating that as the concentration of permeation enhancer is increased the drug diffusion and permeability was increased. Another explanation for this occurred due the concentration of RL 100 (R3), HPMC-4KM (R9) and EC (R12) release liners (Figure 7), when they contact with aqueous media they swell 


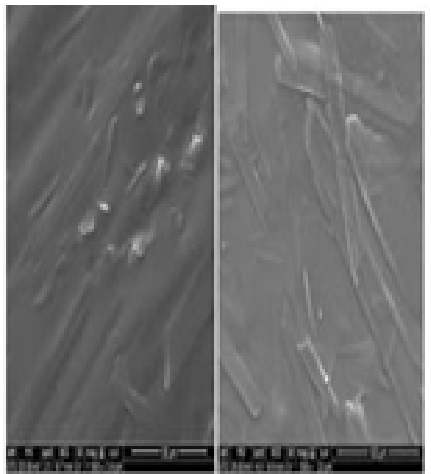

a

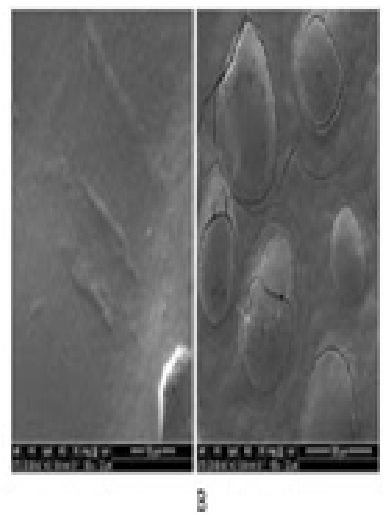

政
Figure 1: Typical SEM Photographs of TC3 (A) and TC12 (B). Scale Bar 300X $-100 \mu \mathrm{m}$.

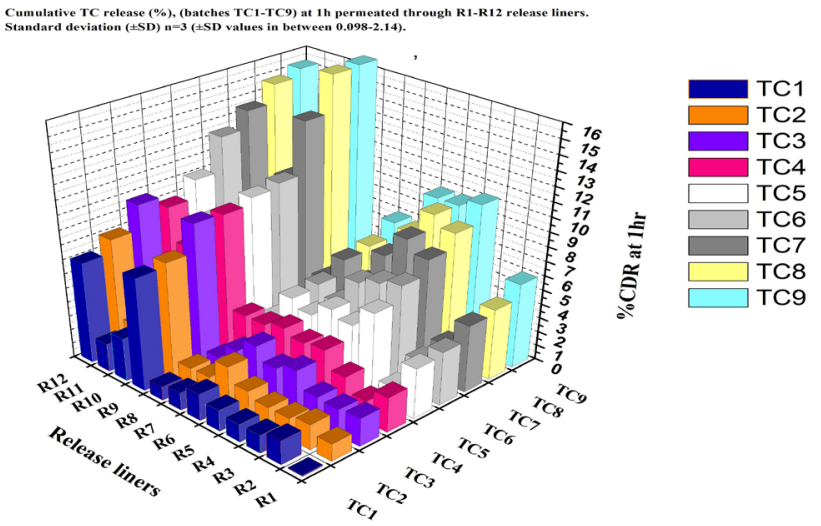

Figure 2: Cumulative TC release (\%), (batches TC1-TC9) at $1 \mathrm{~h}$ permeated through R1-R12 release liners. Standard deviation $( \pm S D) n=3$ ( \pm SD values in between 0.098-2.14).

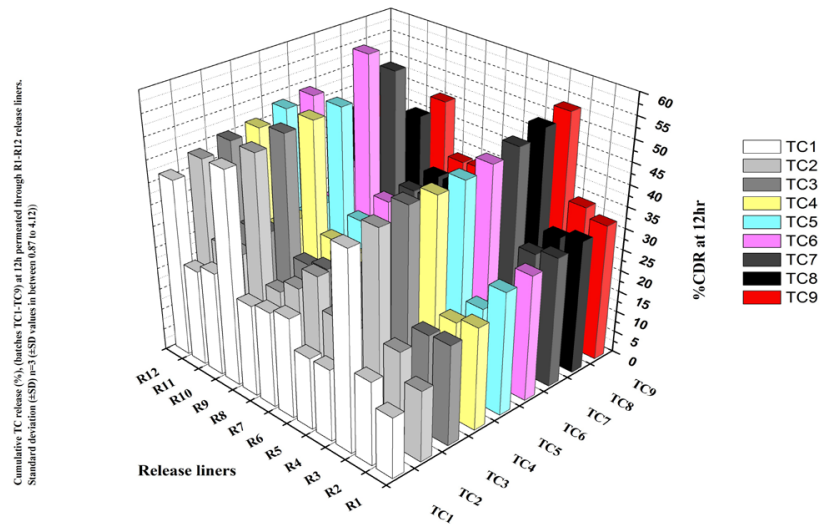

Figure 3: Cumulative TC release (\%), (batches TC1-TC9) at $12 \mathrm{hr}$ permeated through R1-R12 release liners. Standard deviation $( \pm S D) n=3$ ( \pm SD values in between 0.87-4.12).

formation of simple micro pores. However, the gel containing high concentration of DMSO could create the micro pores on the surface of the release liner and finally increase in the diffusion (permeation) of the drug from the formulation was concluded. The highest J. Flux and permeation was found in the order of

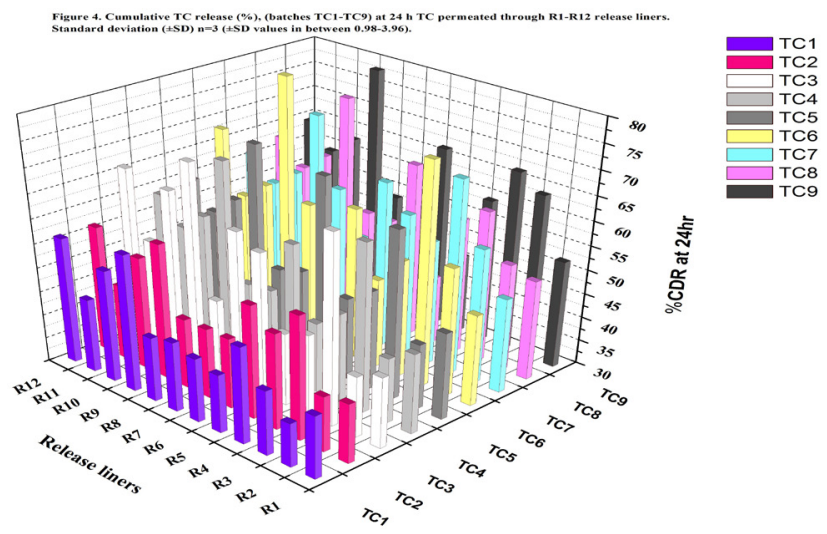

Figure 4: Cumulative TC release (\%), (batches TC1-TC9) at 24 $\mathrm{h}$ TC permeated through R1-R12 release liners.

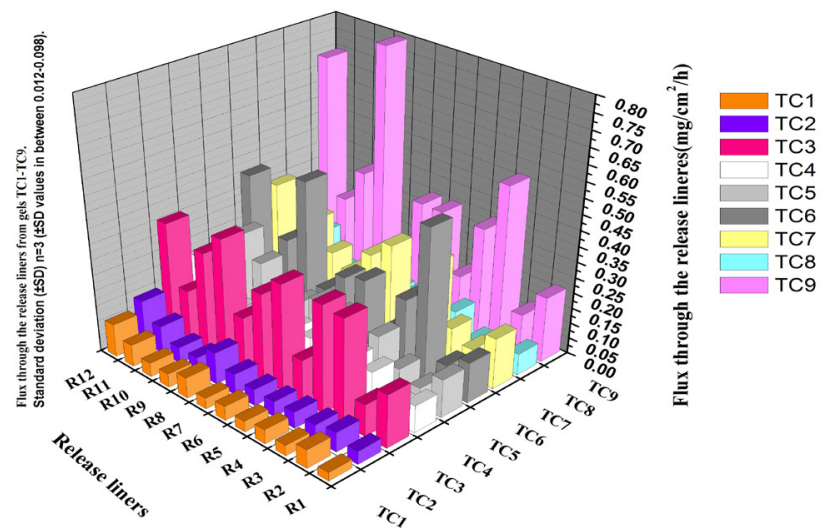

Figure 5: Flux through the release liners from gels TC1-TC9. Standard deviation $( \pm S D) n=3( \pm S D$ values in between 0.012 0.098).

Drug Permeability coefficient $\left(\mathrm{mg} / \mathrm{cm}^{2} / \mathrm{h}\right)$ through release liners from gels TC1-TC Standard deviation $( \pm \mathrm{SD}) \mathrm{n}=3$ ( $(\mathrm{SD}$ values in between $0.002-0.006)$.

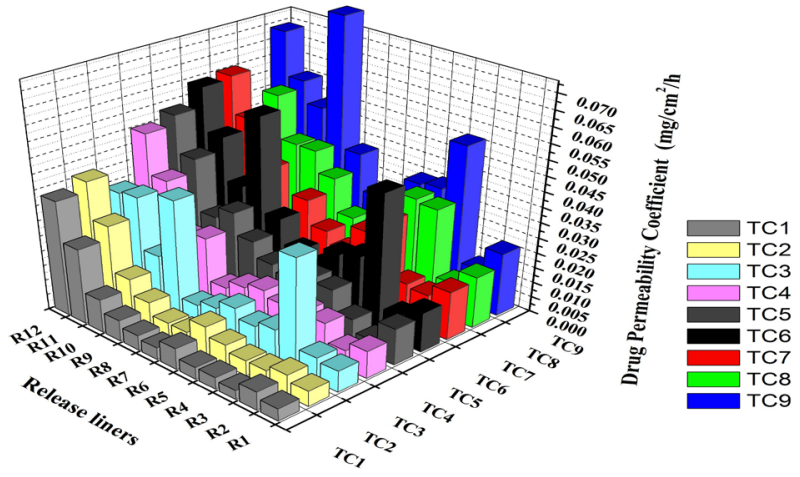

Figure 6: Drug Permeability coefficient $(\mathrm{mg} / \mathrm{cm} 2 / \mathrm{h})$ through release liners from gels TC1-TC9. Standard deviation $( \pm S D)$ $n=3( \pm S D$ values in between $0.002-0.006)$.

TC7 $>$ TC $3>$ TC6 $>$ TC9 is $0.309 \pm 0.04, \quad 0.359 \pm 0.08$, $0.409 \pm 0.04,0.694\left(\mathrm{mg} / \mathrm{cm}^{2} / \mathrm{h}\right)$ and $0.034 \pm 0.002$, $0.037 \pm 0.004, \quad 0.059 \pm 0.006,0.0694 \pm 0.003, \mathrm{mg} / \mathrm{cm}^{2} / \mathrm{h}$. The lowest flux and permeation was found in the order of $\mathrm{TC} 1>\mathrm{TC} 2>\mathrm{TC} 4>\mathrm{TC} 5>\mathrm{TC} 8$ is $0.027 \pm 0.02$, $0.042 \pm 0.34,0.087 \pm 0.028,0.066 \pm 0.061,0.123 \pm 0.043$, 
$\mathrm{mg} / \mathrm{cm}^{2} / \mathrm{h}$ ) and $0.004 \pm 0.002,0.005 \pm 0.001,0.009 \pm 0.002$, $0.016 \pm 0.005$ and $0.017 \pm 0.004\left(\mathrm{mg} / \mathrm{cm}^{2} / \mathrm{h}\right)$, respectively. We also evaluated the kinetics of the release and fitted the results in to various release models Among the hydrogel formulations, TC3, TC6, TC9 and TC12 fitted well into the zero order release model, showing correlation coefficient values between 0.985to 0.996, while TC1, TC2, TC4, TC5, TC7 and TC8 hydrogels best fitted with first-order release kinetics. Hixon-Crowell and Highuchi models were also applied to the release data. This showed a higher $R^{2}$ values for Highuchi than Hixon-Crowell model for all formulations. Additionally, it seemed that the data of drug release of all batches followed a diffusion rate-controlled mechanism. Another tested model of the release was Korsmeyer-Peppas. Considering that, we found that in general the of TC3, TC6, TC9 and TC12 formulations were $>0.785$. This indicates that the release mechanisms of these formulations follow the zero order model, while for TC1, TC2, TC4, TC5, TC7 and TC8 hydrogels the values were between 0.624 to 0.735 implying a non-Fickian diffusion release mechanism.

Next we used an in vitro skin permeation study using rate skin and Keshary-Chien type diffusion cells. Based on our previous release results, we selected R3, R6, R12 membranes and evaluated the most efficient prepared gel formulations (TC3, TC6 and TC9) using this in vitro approach. Moreover the TC3, TC6 and TC9 gel formulations have a combination of hydrophilic a hydrophobic polymers characteristic. We determined the cumulative drug release percentage ( $\%$ CDR), J Flux and permeation coefficient $(\mathrm{Kp})$ values of different hydro$\mathrm{gel} / \mathrm{membrane}$ combinations. Among different formulations, we found that TC3 gel versus R3, R6 and R12 membranes had a \%CDR of $0.79 \pm 1.2,3.05 \pm 0.98$ and $12.38 \pm 0.67, \mathrm{mg} / \mathrm{cm}^{2} / \mathrm{h}$, respectively, at $1 \mathrm{~h}$ evaluation.

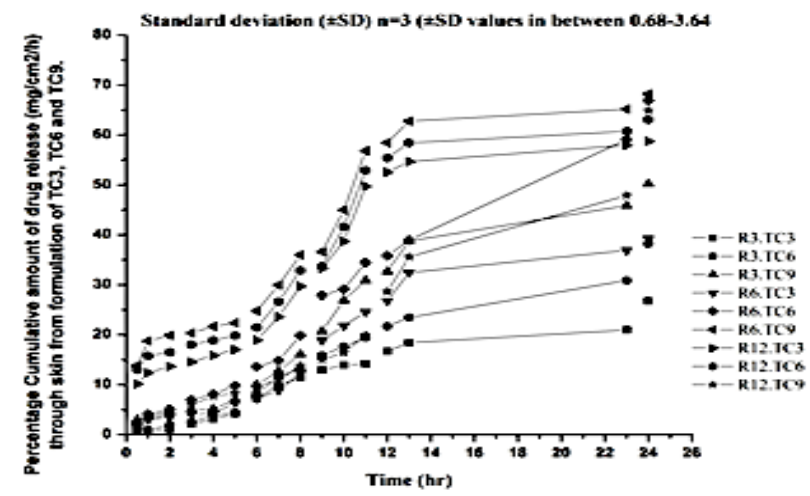

Figure 7: Percentage Cumulative amount of drug release ( $\mathrm{mg} /$ $\mathrm{cm}^{2} / \mathrm{h}$ ) through skin from formulation of TC3, TC6 and TC9 with release liner $R 3, R 9$ and $R 12$. Standard deviation ( $(\mathrm{SD})$ $n=3$ ( \pm SD values in between $0.002-0.006$ ).
At $12 \mathrm{hr}, 16.78 \pm 1.4,26.78 \pm 1.64$ to $52.52 \pm 2.34$ and end of $24 \mathrm{hr}$ it was $26.75 \pm 2.62,39.24 \pm 2.8$ and $58.74 \pm 3.6$ $\mathrm{mg} / \mathrm{cm}^{2} / \mathrm{h}$.

TC6 gel versus R3, R6 and R12 membranes had a $\%$ CDR of $0.98 \pm 0.65,4.1 \pm 0.56$ to $15.7 \pm 0.46 \mathrm{mg} / \mathrm{cm}^{2} / \mathrm{h}$ at $1 \mathrm{~h}$ evaluation, however, the same formulation at $12 \mathrm{it}$ was found that $21.68 \pm 1.48$ to $55.50 \pm 0.98$, at the end of $24 \mathrm{hr}$ is $21.68 \pm 0.83,35.81 \pm 2.34$ and $63.08 \mathrm{mg} / \mathrm{cm}^{2} / \mathrm{h}$. Moreover, \%CDR of TC9 versus R3, R6 and R12 membranes at $1 \mathrm{~h}$ evaluation were $3.5 \pm 0.42,18.75 \pm 0.64$ and $3.09 \mathrm{mg} / \mathrm{h} / \mathrm{cm}^{2}$, at $12 \mathrm{hr}$ it was found that $32.67 \pm 0.97$, $58.73 \pm 2.44$ and $45.65 \mathrm{mg} / \mathrm{h} / \mathrm{cm}^{2}$ and also at $24 \mathrm{hr}$ the valves were $50.21 \pm 0.95,68.24 \pm 0.54$ and $64.89 \mathrm{mg} / \mathrm{h} /$ $\mathrm{cm}^{2}$, respectively. This study indicate that Tamoxifen containing gel successfully permeated skin through rate controlling membranes with the effect of enhancer DMSO induced the formation of transient "water pores" in the cell membrane, consequently increases the fluidity of the bilayer. ${ }^{15-17}$

Figure 8 shows the steady state skin J. Flux of TC3 gel versus R3, R6 and R12 membranes were 0.079 \pm 0.02 , $0.035 \pm 0.04$ and $0.123 \pm 0.01 \mathrm{mg} / \mathrm{cm}^{2} / \mathrm{h}$, TC6 gel versus R3, R6 and R12 membranes were $0.209 \pm 0.04$, $0.244 \pm 0.03$ and $0.245 \pm 0.01 \mathrm{mg} / \mathrm{cm}^{2} / \mathrm{h}, \mathrm{J}$. Flux of TC3 gel versus same membranes were $0.441 \pm 0.04$, $0.663 \pm 0.05$ and $0.558 \pm 0.06 \mathrm{mg} / \mathrm{cm}^{2} / \mathrm{h}$.

The skin permeation coefficient of TC3, TC6 and TC9 combined with R3, R6 and R12 membranes are reported in Figure 9. The obtained data was TC3 versus R3, R6 and R12 were $0.008 \pm 0.002,0.009 \pm 0.003$ and $0.013 \mathrm{mg} / \mathrm{cm}^{2} / \mathrm{h}$. In case of TC6 versus R3, R6 and R12 were $0.022 \pm 0.001,0.025 \pm 0.004$ and $0.026 \pm 0.001$ and $\mathrm{T} 9$ versus $\mathrm{R} 3, \mathrm{R} 6$ and $\mathrm{R} 12$ determined valves were $0.004 \pm 0.001,0.066 \pm 0.002$ and $0.056 \pm 0.003 \mathrm{mg} /$ $\mathrm{cm}^{2} / \mathrm{h}$, respectively.

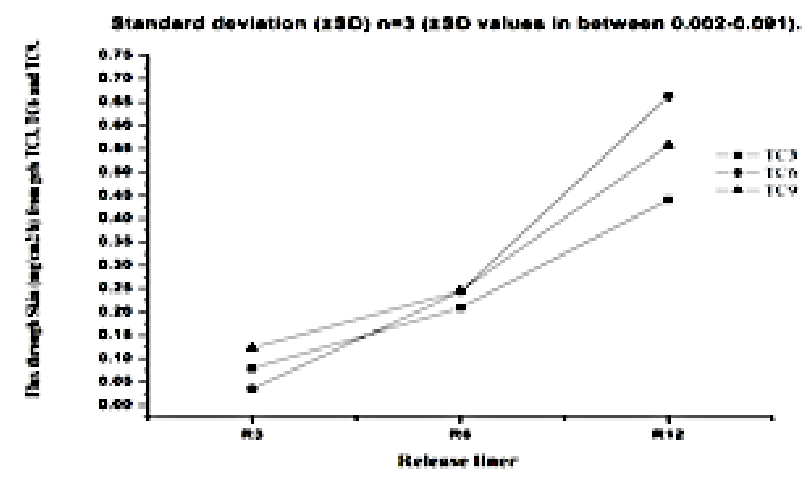

Figure 8: Flux through Skin $\left(\mathrm{mg} / \mathrm{cm}^{2} / \mathrm{h}\right)$ from gels TC3, TC6 and TC9. Standard deviation $( \pm \mathrm{SD}) n=3( \pm \mathrm{SD}$ values in between 0.002-0.091). 


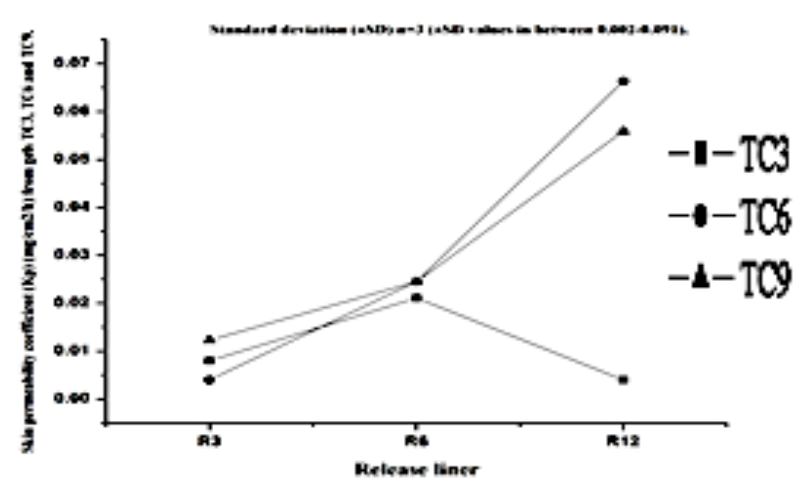

Figure 9: Skin permeability coefficient $(\mathrm{Kp})\left(\mathrm{mg} / \mathrm{cm}^{2} / \mathrm{h}\right)$ from gels TC3, TC6 and TC9. Standard deviation $( \pm S D) n=3( \pm S D$ values in between $0.002-0.091$ ).

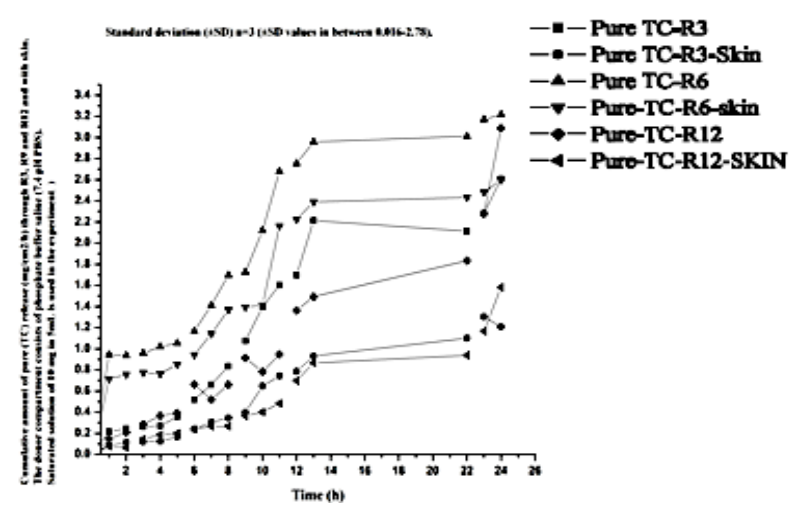

Figure 10: Cumulative amount of pure (TC) release $\left(\mathrm{mg} / \mathrm{cm}^{2} / \mathrm{h}\right)$ through R3, R9 and R12 and with skin. The donor compartment consists of phosphate buffer saline (7.4 pH PBS). Saturated solution of $10 \mathrm{mg}$ in $5 \mathrm{~mL}$ is used in the experiment). Standard deviation $( \pm S D) n=3( \pm S D$ values in between 0.016-2.78).

Figure 10 show the pure Tamoxifen citrate shows the cumulative amount of drug permeated through release membranes and pure Tamoxifen citrate skin permeation through rate controlling membranes. The obtained data revealed that the $2.66 \pm 0.92$ to $3.27 \pm 0.65$ $\mathrm{mg} / \mathrm{cm}^{2} / \mathrm{h}$ of dug permeated across the rate controlling membranes (R3, R6 and R12) at the end of $24 \mathrm{hr}$. Similarly, only $1.24 \pm 0.05$ to $2.56 \pm 0.09 \mathrm{mg} / \mathrm{cm}^{2} / \mathrm{h}$ of dug permeated across the skin. From these experiments we concluded that solubility of drug in aqueous solution is the major barrier and solubility of tamoxifen base is $0.0004 \mathrm{mg} / \mathrm{ml}$ and its salt form is $2.0-3.0 \mathrm{mg} / \mathrm{ml}$. The salt form of drug is partially soluble but less partitioning of the drug through the skin and research group reported that, the Tamoxifen is an ideal candidate for TDDS; however transdermal delivery of moderately large molecules such as tamoxifen (MW 563.65) is often extremely difficult, if not impossible, without the assistance of penetration enhancers. Hence, our investigated results therefore suggest that TC loaded HPMC-K100 and Carbopol-934 gels and rate controlling membranes have may serve as effective drug carrier and a useful anticancer platform. In our present study the lag times seen are relatively short indicating that steady state flux is achieved rapidly and the rapid uptake from transdermal reservoir. A common release lag time was found for the tested formulations ranging between 28 to $36 \mathrm{~min}$. This might indicate that the variation in the TC cumulative release, J. Flux and permeability coefficient of every formulation depend upon the donor compartment and the condition of the skin. Also the drug concentration gradient in biological membrane can significantly contribute in the release behaviour from the TDDS. This is in fact due to the very complex and heterogeneous nature of the biological membranes. ${ }^{30,32}$ The analysis of TC content in TC3, TC6 and TC9 gel formulations confirmed a full recovery of the drug from the gel network. We found that the TC released content was about $98-99 \% \pm 2.8$ in these formulations. Furthermore, TC3, TC6 and TC9 subjected to the release kinetics modelling (data not shown) revealed that in general the TC release was mainly governed by the passive Fickian diffusion mechanism $\left(R^{2}\right.$, regression coefficient $\left.=0.9876\right)$ and zero order kinetic model with the correlation coefficient values between 0.993 and 0.996 .

\section{CONCLUSION}

In this study we evaluated by prepared membranes by Eudragit, HPMC4K and EC polymers for release controlling of TC from the hydrogel sin a TDDS. The obtained results revealed that reservoir-type TDDS of TC successfully can be prepared by a combination of HPMC-K100 and Carbopol 934. The suitable and transparent films of Eudragit RS100, RL 100 HPMC K4M and EC were developed. The physicochemical studies of the prepared gels revealed a non-Newtonian (viscoelastic) behavior of these gels. The in vitro and skin permeation results of the prepared TC-containing gels could be considered suitable in term of transdermal delivery application. Based on these results, TCcontaining gel formulations showed a zero order and non-Fickian diffusion release mechanism. Additionally, the $\mathrm{J}_{\mathrm{ss}}$ Flux result of the formulations was found to be highly dependent on the concentration in a nonlinear fashion. TDDS composed of a combination of the selected polymers showed an excellent drug release properties through RS100, HPMC-K4 and EC film as the rate-controlling membranes (release liners). The optimized TDDS can be a promising candidate for further studies in human cadaver skin and in vivo. These will pave the way to eventually prepare a convenient trans- 
dermal delivery approach in hormonal therapy of breast cancer patients.

\section{ACKNOWLEDGEMENT}

This research was supported by Deanship of Scientific Research, King Faisal University, Al-Ahsa, Saudi Arabia. (Grant Number: 160109)

\section{CONFLICT OF INTEREST}

The authors declare that there are no conflicts of interest.

\section{ABBREVIATIONS}

TDDS: Transdermal Drug Delivery System; EC: Ethyl Cellulose; FDA: Food and Drug Administration; SC: Stratum Corneum; DMSO: Dimethyl Sulfoxide; DBH: Dibutyl phthalate.

\section{REFERENCES}

1. Regan MM, Francis PA, Pagani O, et al. Absolute benefit of adjuvant endocrine therapies for premenopausal women with hormone receptor-positive, human epidermal growth factor receptor 2-negative early breast cancer: TEXT and SOFT trials. Journal of Clinical Oncology. 2016;34(19):2221.

2. Kalvimoorthi V, Rajasekaran M, Rajan S, et al. Transdermal Drug Delivery System: an Overview. International Journal of Pharmaceutical and Chemical Sciences. 2015;4: 90.

3. Elias PM. Epidermal lipids, barrier function and desquamation. Journal of Investigative Dermatology. 1983;80.

4. Elias PM, Menon GK. Structural and lipid biochemical correlates of the epidermal permeability barrier. Advances in Lipid Research: Elsevier. 1991;24:1-26

5. Choy YB, Prausnitz MR. The rule of five for non-oral routes of drug delivery: ophthalmic, inhalation and transdermal. Pharmaceutical Research. 2011;28(5):943-8.

6. Dancik $\mathrm{Y}$, Miller MA, Jaworska $\mathrm{J}$, et al. Design and performance of a spreadsheet-based model for estimating bioavailability of chemicals from dermal exposure. Advanced Drug Delivery Reviews. 2013;65(2):221-36.

7. Chandak AR, Prasad VPR. Eudragit-based transdermal delivery system of pentazocine: Physico-chemical, in vitro and in vivo evaluations. Pharmaceutical Development and Technology. 2010;15(3):296-304.

8. Aisha AFA, Abdulmajid AMS, Ismail Z, et al. Development of Polymeric Nanoparticles of Garcinia mangostana Xanthones in Eudragit RL100/RS100 for Anti-Colon Cancer Drug Delivery. Journal of Nanomaterials 2015;2015:12. DOI: 10.1155/2015/701979

9. Roberts GP, Barnes HA. New measurements of the flow-curves for Carbopol dispersions without slip artefacts. Rheologica Acta. 2001;40(5): 499-503.

10. Valenta C, Auner BG. The use of polymers for dermal and transdermal delivery. European Journal of Pharmaceutics and Biopharmaceutics. 2004;58(2):279-89.

11. Güngör S, Erdal MS, Özsoy Y. Plasticizers in transdermal drug delivery systems. Recent Advances in Plasticizers: Intech Open. 2012.

12. Kathe K, Kathpalia H. Film forming systems for topical and transdermal drug delivery. Asian Journal of Pharmaceutical Sciences. 2017;12(6):487-97. DOI: https://doi.org/10.1016/j.ajps.2017.07.004
13. Ghaffarian R, Muro S. Models and methods to evaluate transport of drug delivery systems across cellular barriers. JOVE. 2013;17(80):e50638.

14. Kaplun-Frischoff $Y$, Touitou E. Testosterone skin permeation enhancement by menthol through formation of eutectic with drug and interaction with skin lipids. Journal of Pharmaceutical Sciences. 1997;86(12):1394-19.

15. Ongpipattanakul B, Burnette RR, Potts RO, et al. Evidence that oleic acid exists in a separate phase within stratum corneum lipids. Pharmaceutical Research. 1991;8(3):350-4

16. Barry B, Bennett S. Effect of penetration enhancers on the permeation of mannitol, hydrocortisone and progesterone through human skin. Journal of Pharmacy and Pharmacology. 1987;39(7):535-46.

17. Mitriaikina S, Muller-Goymann C. Synergetic effects of Isopropyl Alcohol (IPA) and Isopropyl Myristate (IPM) on the permeation of betamethasone17-valerate from semisolid Pharmacopoeia bases. Journal of Drug Delivery Science and Technology. 2007;17(5):1-8.

18. Marren K. Dimethyl Sulfoxide: an Effective Penetration Enhancer for Topical Administration of NSAIDs. The Physician and Sportsmedicine. 2011;39(3):7582. DOI: 10.3810/psm.2011.09.1923

19. Barry B, Williams A. Penetration enhancers. Adv Drug Deliv Rev. 2003;56:60318.

20. Gurtovenko AA, Anwar J. Modulating the structure and properties of cell membranes: the molecular mechanism of action of dimethyl sulfoxide. The Journal of Physical Chemistry B. 2007;111(35):10453-60.

21. Gurtovenko AA, Anwar J, Vattulainen I. Defect-mediated trafficking across cell membranes: insights from in silico modeling. Chemical Reviews. 2010;110(10):6077-103.

22. Hiremath J, Rudani C, Domb A, et al. Preparation and in vitro characterization of poly (sebacic acid-co-ricinoleic acid)-based tamoxifen citrate-loaded microparticles for breast cancer. Journal of Applied Polymer Science. 2012;124(6):4747-54.

23. Bachhav $Y$, Patravale $V$. Formulation of meloxicam gel for topical application: in vitro and in vivo evaluation. Acta Pharmaceutica. 2010;60(2):153-63.

24. Hiremath J, Rudani C, Suthar R, et al. Tamoxifen citrate loaded biodegradable poly (sebacic acid-co-ricinoleic acid) microparticles, in vitro characterization. Journal of Drug Delivery Science and Technology. 2011;21(5):417-22.

25. Li GL, DerGeest RV, Chanet L, et al. In vitro iontophoresis of R-apomorphine across human stratum corneum: structure-transport relationship of penetration enhancement. Journal of Controlled Release. 2002;84(1-2):49-57.

26. Yang $\mathrm{Z}$, Teng $\mathrm{Y}$, Wang $\mathrm{H}$, et al. Enhancement of skin permeation of bufalin by limonene via reservoir type transdermal patch: formulation design and biopharmaceutical evaluation. International Journal of Pharmaceutics. 2013;447(1-2):231-40.

27. Baviskar DT, Parik VB, Jain DJ. Development of Matrix-type transdermal delivery of lornoxicam: in vitro evaluation and pharmacodynamic and pharmacokinetic studies in albino rats. PDA Journal of Pharmaceutical Science and Technology. 2013;67(1):9-22.

28. Dey SK, De PK, Sen T, et al. Formulation and in vitro evaluation of transdermal matrix patches of Diclofenac sodium. Journal of Pharmacy Research. 2011;4(10):3593-6.

29. Mutalik S, Udupa N. Glibenclamide transdermal patches: physicochemical, pharmacodynamic and pharmacokinetic evaluations. Journal of Pharmaceutical Sciences. 2004;93(6):1577-94.

30. Zorin S, Kuylenstierna $\mathrm{F}$, Thulin $\mathrm{H}$. In vitro test of nicotine's permeability through human skin: risk evaluation and safety aspects. Annals of Occupational Hygiene. 1999;43(6):405-13

31. Dangre PV, Godbole MD, Ingale PV, et al. Improved dissolution and bioavailability of eprosartan mesylate formulated as solid dispersions using conventional methods. Indian J Pharm Edu Res. 2016;50(3):S209-17.

32. Babu R, Pandit J. Effect of penetration enhancers on the release and skin permeation of bupranolol from reservoir-type transdermal delivery systems. International Journal of Pharmaceutics. 2005;288(2):325-34. 
PICTORIAL ABSTRACT

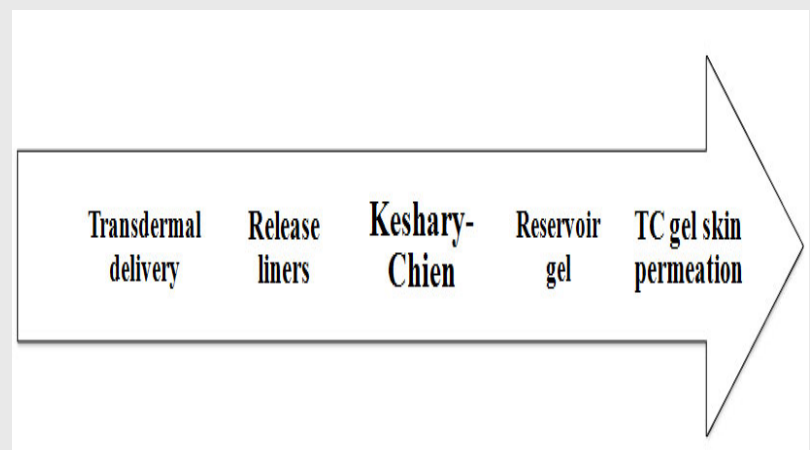

\section{SUMMARY}

The present investigation, topical gel TC was prepared using HPMC K4M and Carbopol 934 as gelling agents in different proportions with Dimethyl Sulfoxide (DMSO) as a penetration enhancer. TC gels were subjected for the physicochemical parameters such as $\mathrm{pH}$, viscosity, spreaddability. The TC gel diffusion was conducted by using rate controlling membranes (release liner). The TC gel skin permeation was investigated by using excised rat skin as a barrier.

\section{About Author}

Dr. Sree Harsha received his (ranked top 5) Master of Pharmacy Degree and subsequently earned a doctorate in Pharmaceutics from Rajiv Gandhi University of Health Sciences, Bangalore, India in 2006. He came to King Faisal University in 2007 as an assistant professor in the Department of Pharmaceutical Sciences, bringing with him several years' worth of teaching experience in fundamentals of pharmaceutics and drug delivery systems. He was actively participated in Accreditation Council of Pharmacy Education (ACPE) and Canadian Council for the Accreditation of Pharmacy Programs (CCAPP). His primary area of focus is pharmaceutical technology and novel/ targeted drug delivery systems. For this research, he received grants (20 number) from Deanship of Scientific research, King Faisal University. The author contributed so far to 52 peer-reviewed full papers on a variety of topics in lung targeting, topical drug delivery, and mucoadhesive drug delivery systems, He has contributed in writing a book chapter titled "Targeted Drug Delivery System" and "Microspheres" in Textbook of Industrial Pharmacy, Publisher-Orient Longman Private Ltd. In addition, he is an Ad-hoc reviewer for scientific journals. He has attended many seminars and Workshop both national and international on Pharmaceutical Technology and Public health issues.

Cite this article: SreeHarsha N, Hiremath JG, Rawre BK, Puttaswamy N, Al-Dhubiab BE, Venugopala KN, Akrawi $\mathrm{SH}$, Meravanige G, Attimarad M, Nair AB. Formulation and Evaluation of Tamoxifen Citrate Loaded Transdermal Reservoir Gel Drug Delivery Systems. Indian J of Pharmaceutical Education and Research. 2019;53(4s):s596-s606. 